\title{
COLECCIÓN Y EVALUACIÓN DE GERMOPLASMA DE (Mauritia flexuosa L. F) AGUAJE EN LAAMAZONÍA PERUANA
}

\author{
COLLECTION AND EVALUATION OF AGUAJE (Mauritia flexuosa L. F.) GERMPLASM IN \\ THE PERUVIANAMAZON
}

Agustín Gonzales Coral $^{1}$, Anita R. Jarama Vilcarromero² ${ }^{2}$ Golberth Chuquival Tuesta², Ruby Vargas Isla²

\section{RESUMEN}

El Instituto de Investigaciones de la Amazonía Peruana (IIAP) ha iniciado un programa de mejoramiento genético de Mauritia flexuosa L. f. aguaje, mediante colecciones de germoplasma, en el departamento de Loreto, entre los años 1999 y 2001. Se colectaron 30 entradas en poblaciones naturales, chacras de agricultores, y huertos familiares, en los distritos de Sapuena y Jenaro Herrera (Requena), Parinari (Loreto), Lagunas (Alto Amazonas), Santa María, San Juan, Punchana, Indiana e Iquitos (Maynas). Los registros de las colecciones se realizaron con el uso de descriptores de pasaporte propuesto por el descriptor de frutas tropicales.

El banco de germoplasma cuenta con 30 introducciones, con 33 individuos cada uno, y está situado en el Centro de Investigaciones Allpahuayo, del Instituto de Investigaciones de la Amazonía Peruana (IIAP), localizada entre los $\mathrm{km} 25.5$ y 28.5 de la carretera Iquitos - Nauta.

En la evaluación de infrutescencias se encontraron los valores promedios de: longitud de pedúnculo $94 \mathrm{~cm}$, longitud de raquis $186 \mathrm{~cm}$, número de raquillas 40 , longitud de raquillas $89 \mathrm{~cm}$, frutos por raquillas $14 \mathrm{y}$ frutos por racimo 548.

Respecto a descriptores de los frutos se encontraron valores promedio de: largo de fruto $5.37 \mathrm{~cm} \mathrm{y} \mathrm{ancho} 3.90 \mathrm{~cm}$, largo de semilla $4.00 \mathrm{~cm}$ y ancho $2.91 \mathrm{~cm}$, peso del fruto $59.68 \mathrm{~g}$, peso de semilla $27.32 \mathrm{~g}$, peso de cáscara 11.15 g, peso de pulpa $15.55 \mathrm{~g}$, peso de episperma $4.70 \mathrm{~g}$. La composición porcentual de frutos fue: semilla $46.29 \%$, pulpa $26.41 \%$, cáscara $19.27 \%$ y epispermo $7.91 \%$.

Palabras clave: Mauritia flexuosa, aguaje, germoplasma, evaluación, conservación, Amazonía peruana, Allpahuayo.

\begin{abstract}
The Peruvian Amazonia Research Institute (IIAP) has launched a genetically improvement program for Mauritia flexuosa L.f. aguaje, by implementing germplasm collections in the Loreto region between the years 1999 to 2001 . Thirty registers were collected from several places: natural populations, farmhouses, and family-owned arboretum. The collections took place in the districts of Sapuena, Jenaro Herrera (Requena); Parinari (Loreto); Lagunas (Alto Amazonas); Santa María, San Juan, Punchana, Indiana e Iquitos (Maynas). Passport descriptors, as proposed by the tropical fruit descriptor, were used during the collection.

The germplasm bank has 30 introductions, each introduction has 33 specimens. This bank is located in the Allpahuayo Research Centre, operated by the Peruvian Amazonia Research Institute, located between the km 22.5 and 28.5 on the Iquitos-Nauta highway.
\end{abstract}

1 Instituto de Investigaciones de la Amazonía Peruana, Programa de Biodiversidad. Correo electrónico: agonzales@iiap.org.pe

2 Universidad Nacional de la Amazonía Peruana - UNAP, Facultad de Agronomía, Iquitos, Perú. 
During the evaluation of the infructescence the following average values, were found: peduncle length of $94 \mathrm{~cm}$, rachis length of $186 \mathrm{~cm}, 40$ rachille, rachille lengths of $89 \mathrm{~cm}$, fruits per rachille 14 and fruits per cluster 548 .

For the fruit descriptors, average values found were: fruit length $5.37 \mathrm{~cm}$ and width $3.90 \mathrm{~cm}$; seed length $4.00 \mathrm{~cm}$ and width $2.91 \mathrm{~cm}$; fruit weight $59.68 \mathrm{~g}$; seed weight $27.32 \mathrm{~g}$; skin weight $11.15 \mathrm{~g}$; pulp weight $15.55 \mathrm{~g}$; episperm weight $4.70 \mathrm{~g}$. The porcentage fruit composition for the fruits were: seed $46.29 \%$, pulp $26.41 \%$, skin $19.27 \%$ and episperm $7.91 \%$.

Keywords: Aguaje, Mauritia flexuosa, germplasm, evaluation, conservation, peruvian Amazonia, Allpahuayo.

\section{INTRODUCCIÓN}

Una de las fuentes alimenticias más valiosas en la Amazonía la constituyen las palmeras, que son consideradas como el grupo de plantas de mayor importancia y abundancia en el geotrópico. Entre ellas el aguaje (Maurutia flexuosa L.f.) es la palmera, con mayor significación económica en la Región Loreto, la cual es intensamente explotada al estado natural (Valdivia, 1995). Su aprovechamiento inadecuado mediante la tala de la planta es perjudicial para el ecosistema, pues carece de sostenibilidad y tiene un impacto marcadamente negativo en los bosques, la gente y la fauna de la región (Penn, 1999).

Las posibilidades de uso de este fruto son diversas. Entre las más importantes tenemos: como alimento fresco, para la extracción de aceites y elaboración de harina. Otras partes de la planta también son aprovechadas como materiales de construcción, crisnejas para techos, fibras, combustible, producción de larvas de Rynchosphorus palmarum (Curculionidae) conocida como suri y consumida por la población local, herramientas, medicina, bebidas, licores, entre otros (Padoch, 1986) (Mejía, 1986) (Penn, 1999).

Similar importancia tiene este fruto para la fauna neotropical, ya que es un recurso alimenticio principal para los ungulados terrestres amazónicos, tales como el tapir o sachavaca (Tapirus terrestris) y el sajino de collar blanco (Tayassu pecarie), considerados como los principales animales de caza de la Amazonía. No obstante, la tala de la planta para la cosecha del fruto reduce considerablemente el sustento de estos animales, que se ven forzados a emigrar como resultado de la escasez de alimento (Penn, 1999). Las investigaciones relacionadas al tema señalan que estos ungulados se encuentran con mayor frecuencia en los bosques donde existe el aguaje. Los ungulados desempeñan una función importante en la propagación de esta palmera, ya que por medio de sus escupitajos dispersan semillas (Valdivia, 1995).

La potencial importancia industrial del aguaje deriva de sus buenas características organolépticas y de su composición química rica en oligoelementos y vitaminas. Por otro lado, este fruto ostenta cifras importantes en las estadísticas de consumo, y su venta proporciona estimables ingresos para cientos de hogares de Iquitos (Domínguez, 1985).

Antes de su explotación destructiva, las vastas áreas de rodales naturales de aguaje podrían haber aportado un gran suministro de frutos para el mercado; sin embargo, la especie por su condición de planta dioica y de porte alto, trepar el estípite de las plantas hembras resulta difícil y hasta peligroso, razón por la cual el tronco es cortado para obtener los racimos (Penn, 1999). Debido a esto, el número de árboles hembras se ha reducido enormemente, teniendo preponderancia de plantas masculinas en la mayor parte de aguajales cerca de Iquitos y aun en lugares relativamente aislados.

La urgente necesidad de un manejo sostenible del aguaje en la Amazonía peruana ha recibido considerables atenciones en las últimas dos décadas, y las instituciones relacionadas con la investigación han establecido plantaciones de aguaje en Loreto.

La plaga más importante parece ser la Eupalamides cyparissias, cuyas larvas atacan al pedúnculo, caquis, raquillas, pecíolo y raramente al estípite. La actividad de la larva es reconocida externamente por la presencia de excreciones cerca de los orificios de alimentación y por las secreciones gomosas emitidas por la planta como una 
respuesta fisiológica al ataque. Esta larva produce orificios sinuosos que causan la senescencia de la parte reproductiva, y la pérdida de flores y frutos (Delgado \& Couturier, 2003).

La colección, conservación, caracterización, selección y propagación de germoplasma pretende la conservación del recurso y la obtención de ecotipos seleccionados, para manejo de futuras plantaciones, lo que permitirá diversificar la producción y disminuir la presión sobre las poblaciones naturales de la especie.

\section{MATERIAL Y MÉTODO}

Las colecciones se llevaron a cabo en el departamento de Loreto, cuencas de los ríos Marañón, Nanay, Ucayali, Itaya, Huallaga y lugares aledaños a la ciudad de Iquitos.

Las fuentes de colección fueron poblaciones naturales (aguajales), chacras y huertos de agricultores. Se recolectaron directamente de las plantas matrices, frutos y semillas, que nos permitan tener la cantidad de plantas para el banco ex situ, así como para el registro de mediciones biométricas. La documentación de cada muestra fue registrada en las fichas pasaporte (CIRF- FAO, EMBRAPA, INIA).

Los registros biométricos fueron tomados en el momento de la colecta del fruto, e incluyó los parámetros: longitud del pedúnculo, longitud del raquis, número de raquillas, longitud de raquillas y número de frutos por raquilla. Posteriormente se realizaron las mediciones y pesadas de los frutos (largo y ancho de fruto, largo y ancho de las semillas y composición porcentual de los frutos).

Para la instalación del germoplasma, se ubicó un área en el Centro de Investigaciones Allpahuayo del Instituto de Investigaciones de la Amazonia Peruana, situada a la margen derecha de la carretera Iquitos - Nauta, entre los km 25.8 y 28.8

Se prepararon camas almacigueras de aproximadamente 10 x $1 \mathrm{~m}$, donde se removió la tierra y se mezcló con materia orgánica de bosque. Las semillas fueron colocadas a distanciamientos de 10 x $10 \mathrm{~cm}$ y tapados ligeramente con el sustrato original.

Posteriormente, se preparó un substrato con materia orgánica vegetal y tierra negra en proporción 1:1, el que se llenó en bolsas de color negro con aproximadamente $2 \mathrm{~kg}$ del substrato, donde se colocaron las plántulas entre 5 a $10 \mathrm{~cm}$ de altura después de 3 ó 5 días de emergencia, y allí se mantuvieron por espacio de 60 días.

Para el control de germinación, se registraron: fecha de siembra, número de semillas sembradas, fecha de inicio de germinación, tipo de germinación, número de semillas germinadas, fecha de término de germinación y porcentaje de germinación.

El banco de germoplasma ex situ se instaló en un área de 3 ha, con aproximadamente 6 años de recuperación; se desbrozó toda la vegetación herbácea y arbustiva, dejando temporalmente los árboles mayores de $20 \mathrm{~cm}$ de DAP. El diseño de la plantación es de 3 plantas en triángulo de $2 \mathrm{~m}$, con distancia entre triángulos de $8 \mathrm{~m}$ con un total de 33 muestras por muestra.

Se realizaron hoyos de $30 \times 30 \times 30 \mathrm{~cm}$, poniendo en el interior materia orgánica vegetal y luego se colocaron las plantas, previa retirada de la bolsa negra.

\section{RESULTADOS Y DISCUSIÓN}

Se colectaron hasta 30 entradas, (frutos, semillas y plántulas); las fuentes de colección fueron poblaciones naturales y huertos caseros, fuentes importantes para colecta de germoplasma vegetal (Hawkes, 1976 y Ford Lloyd \& Jackson, 1986), correspondiendo a 23 localidades, 11 distritos, 4 provincias en el departamento de Loreto, Perú. (Anexo 1). 
El banco ex situ situado a la margen derecha de la carretera Iquitos - Nauta, km 25.8, cuenta con 30 accesiones (Chiringa, Juana, Tacshacocha, Shuyal, Samito, Yarina, Shiriara, Itaya 1, Itaya 2, Bagazán, Santo Tomás, Santa Rosa, Quistococha 5, Quistococha 1, Santa Clara, Varillal, Quistococha 4, Quistococha 3, INIA Iquitos, Quistococha 2, Mazán 1 -2, Indiana 1, Lagunas 1, Jenaro Herrera, IIAP Central, Lagunas 2, Lagunas 3-4, Nauta-1, Olaya-1, y San Jorge-1).

El registro inicial de las colecciones se realizó en fichas pasaportes (Machado \& Barbosa 1996) con 27 descriptores: nombre científico, familia, nombre común, colector, $\mathrm{N}^{\circ}$ colector, fecha, material colectado, hábito de crecimiento, color fruto, interés económico, lugar colección, tipo de suelo, relieve, frecuencia relativa, país, departamento, provincia, distrito, latitud, longitud, localidad, código del acceso, y otras observaciones.

Los valores promedio de los descriptores obtenidos de 13 colecciones fueron: en racimos, longitud de pedúnculo $94 \mathrm{~cm}$ (56-120), Villachica (1996), reporta la longitud del pedúnculo entre 60 y $100 \mathrm{~cm}$. Longitud de raquis $186 \mathrm{~cm}$ (154-280), para Villachica (1996) entre 70 y $140 \mathrm{~cm}$. La medida promedio del racimo es de $280 \mathrm{~cm}$, encontrándose entre los 200 y $300 \mathrm{~cm}$ (Flores, 1997). Número de raquillas 40 (26-48); longitud de raquillas $89 \mathrm{~cm}$ (69-115); frutos por raquillas 14 (5-41); frutos por racimo 548 (216-1640) (Anexo 2).

Las evaluaciones de 21 ecotipos con 25 frutos cada uno, dieron los siguientes promedios: El peso promedio del fruto es de 58.08 g (29.20-93.64), valores cercanos a Flores (1997). El peso de la semilla $27.32 \mathrm{~g}$ (13.03-43.24); peso de la cáscara $11.15 \mathrm{~g},(4.05-17.41)$; peso de pulpa $15.55 \mathrm{~g}$ (6.62-29.92); peso de episperma $4.70 \mathrm{~g}$ (13.04-2.42).

El largo de frutos en promedio fue $5.37 \mathrm{~cm}(7.32-3.68)$ y ancho $3.90 \mathrm{~cm}(4.88-2.53)$, registros aproximados a FAO/ CATIE (1983), Flores (1997), Villachica (1996), con longitudes que van entre 5 a $7 \mathrm{~cm}$ de largo y 4 a $5 \mathrm{~cm}$. El ANVA, el carácter largo de fruto muestra alta significancia $(\mathrm{fc}=599.50)$, coeficiente de variabilidad (C.V. $=1.42)$. Para el carácter ancho de fruto muestra alta significancia estadística $(\mathrm{fc}=249.71)$, y coeficiente de variabilidad (C. V. 1.42) (Anexo 3).

Largo de semillas $4,00 \mathrm{~cm}(5.21-2.87)$ y ancho $2.91 \mathrm{~cm},(3.60-2.39)$. Se determinó alta significancia estadística para el descriptor largo de semilla $(\mathrm{fc}=147.49)$, con C. V. $=2.37$. Igualmente, para el carácter ancho de semilla la significancia estadística fue alta $(\mathrm{fc}=68.44), \mathrm{C} . \mathrm{V} .=2.49($ Anexo 3$)$.

La composición porcentual de los frutos fue: semilla $46.29 \%$ del peso del fruto (56.03-39.97), cercano a los reportes de Flores (1997), que reporta porcentajes de semilla con relación al fruto entre 40 y 44.50 \%; pulpa, 26.41 $\%$ (31.91-18.38), reportes de Villachica (1996), el mesocarpio corresponde entre 12 y el $13 \%$ del fruto, mientras que Flores (1997) menciona que el mesocarpo corresponde al 10 - $21 \%$ del fruto. La cáscara, $19.27 \%$ (24.53-11.40); el epispermo, $7.91 \%$ (15.04-4.57), resultados muy cercanos a los reportados por FAO/CATIE, 1983. (Figura $1 \mathrm{y}$ Anexo 4).

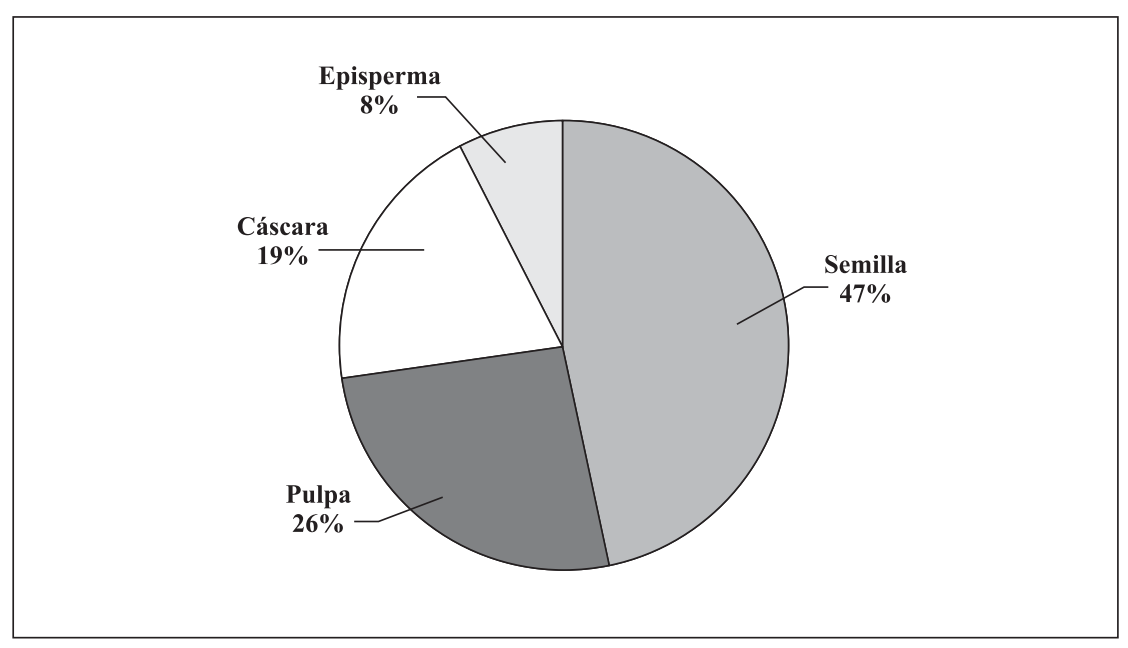

Figura 1. Composición porcentual del fruto de aguaje 
Las semillas tuvieron una germinación muy variada e hipogea, encontrándose entre los ecotipos evaluados el inicio de la germinación en promedio de 62.95 días (40-94), con porcentajes de germinación que van desde $9.09 \%$ hasta $88.18 \%$ Geilfus (1994) registró el inicio de la germinación a los 82 días, alcanzando a los 101 días, $40 \%$ de germinación. En Manaus (Brasil) semillas procedentes de frutos que contenían una semilla, a los 92 días germinaron el $48 \%$ y a los 120 días finales germinaron el $52 \%$. En semillas procedentes de frutos que contenían 2 semillas, a los 55 días germinó el 41\%. Y entre 120 a 150 días finales de germinación, 64\%. (Anexo 5).

\section{AGRADECIMIENTOS}

Los autores expresan su sincero agradecimiento a los especialistas del IIAP: Kember Mejía, Mario Pinedo y César Delgado por las revisiones y sugerencias al presente trabajo.

\section{BIBLIOGRAFÍA}

DELGADO, C; COUTURIER, G. 2003. Relationship between Mauritia flexuosa y Eupalamides cyparissias in the Peruvian Amazon. Palms 47(2):104-106.

DOMÍNGUEZ, A. 1995. Ensayos de procesamiento de pijuayo y aguaje. IIAP. Iquitos, Perú 26 p.

FAO/CATIE. 1983. Situación actual de la investigación y desarrollo en palmeras poco conocidas. Informes por especies y países. Mauritia In: Palmeras poco utilizadas de América Tropical, Turriallba, Costa Rica.

FLORES P. S. 1997. Cultivos de frutales nativos amazónicos, manual para el extensionista. TCA, p. 7-13

FORD-LLOYD, B.; JACKSON, M. 1986. Plant Genetic resources. An introduction to their conservation and use. London: Edward Arnold. 146 p.

GEILFUS. F. 1994. El árbol al servicio del agricultor. Manual de Agroforestería para el desarrollo rural. Vol. 2 Guía de especies. Enda-Caribe. CATIE.

HAWKES, J. 1976. Manual for field collectors: seed crop. Rome. FAO. 34 p.

MACHADO, T. W.B., BARBOSA, C.T. 1996. Coleta de Germoplasma Vegetal: Teoria e Práctica. EMBRAPA. CENARGEN. Brasilia-DF-Brasil. 83 p.

MEJ́A, K. 1986. Utilization of Palms in Eleven Mestizo Villages of the Peruvian Amazon (Ucayali River, Departament of Loreto). Advances in Economic Botaniy 6: 130-136.

PADOCH, C. 1986. Aguaje (Mauritia flexuosa L.f) in the economy of Iquitos, Perú.. Advances in Economic Botaniy 6: 214-224.

PENN, J. W. 1999. The aguaje (Mauritia flexuosa L.f.) : examining role as an agroforestry species in a community conservation project. Gainesville, FL, University of Florida, 1999. 184 p. ilus, tab.

VALDIVIA, I. G. 1995. Variación de la densidad poblacional de Mauritia flexuosa L.f. aguaje, según asociación florística en la quebrada Yanayacu - río Marañón. Universidad Nacional de la Amazonía Peruana, Iquitos, Perú. 73 p.

VILLACHICA, H.1996. Frutales y hortalizas promisorias de la Amazonía SPTTCA, No 44. Lima, Perú. 
Anexo 1. Colección de Mauritia flexuosa L. f, aguaje, en el departamento de Loreto

\begin{tabular}{|c|c|c|c|c|c|}
\hline $\mathbf{N}^{\mathbf{0}}$ & Población & Georeferenciación & Distrito & Provincia & F. de S. \\
\hline 01 & Chiringa & 04³4'40"у 74²7’09" & Parinari & Loreto & $10 / 2000$ \\
\hline 02 & Juana & 043'40"у 74²7’09" & Parinari & Loreto & $10 / 2000$ \\
\hline 03 & Tacshacocha & $04^{\circ} 52^{\prime} 46^{\prime \prime}$ у $74^{\circ} 20^{\prime} 31^{\prime \prime}$ & Parinari & Loreto & $10 / 2000$ \\
\hline 04 & Shuyal & $04^{\circ} 34^{\prime} 40 " \mathrm{y} 74^{\circ} 27^{\prime} 09^{\prime \prime}$ & Parinari & Loreto & $10 / 2000$ \\
\hline 05 & Samito & $03^{\circ} 51^{\prime} 56^{\prime \prime}$ y 73³6'43" & Santa María & Maynas & $10 / 2000$ \\
\hline 06 & Yarina & $03^{\circ} 51$ '38"y 73³5’03" & Santa María & Maynas & $10 / 2000$ \\
\hline 07 & Shiriara & 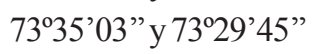 & Santa María & Maynas & $10 / 2000$ \\
\hline 08 & Itaya 1 & $04^{\circ} 14{ }^{\prime} 50 " \mathrm{y} 73^{\circ} 32^{\prime} 59^{\prime \prime}$ & San Juan & Maynas & 09/2001 \\
\hline 09 & Itaya 2 & $04^{\circ} 14^{\prime} 50^{\prime \prime} \mathrm{y} 73^{\circ} 32^{\prime} 59^{\prime \prime}$ & San Juan & Maynas & 09/2001 \\
\hline 10 & Bagazán & $04^{\circ} 43^{\prime} 31^{\prime \prime}$ у 73³2’01" & Sapuena & Requena & $10 / 2001$ \\
\hline 11 & Santo Tomás & $03^{\circ} 47^{\prime} 30^{\prime \prime}$ 7 7318’03" & San Juan & Maynas & $10 / 2001$ \\
\hline 12 & Santa Rosa & $04^{\circ} 58^{\prime} 36^{\prime \prime} 7^{\circ} 46^{\circ} 15^{\prime \prime}$ & Requena & Requena & $10 / 2001$ \\
\hline 13 & Quistococha 5 & $03^{\circ} 47^{\prime} 30^{\prime \prime} \mathrm{y} 73^{\circ} 18^{\prime} 03^{\prime \prime}$ & San Juan & Maynas & $12 / 2001$ \\
\hline 14 & Quistococha 1 & $03^{\circ} 48^{\prime} 54^{\prime \prime}$ у 73¹9'17" & San Juan & Maynas & $12 / 2001$ \\
\hline 15 & Santa Clara & $03^{\circ} 46^{\prime} 32^{\prime \prime}$ у $73^{\circ} 18^{\prime} 18^{\prime \prime}$ & San Juan & Maynas & $12 / 2001$ \\
\hline 16 & Varillal & $03^{\circ} 47^{\prime} 30^{\prime \prime}$ у $73^{\circ} 18^{\prime} 03^{\prime \prime}$ & San Juan & Maynas & $12 / 2001$ \\
\hline 17 & Quistococha 4 & $03^{\circ} 48^{\prime} 50^{\prime \prime}$ у $73^{\circ} 19^{\prime} 08^{\prime \prime}$ & San Juan & Maynas & $12 / 2001$ \\
\hline 18 & Quistococha 3 & $03^{\circ} 48^{\prime} 45^{\prime \prime}$ у 73⒉'53" & San Juan & Maynas & $12 / 2001$ \\
\hline 19 & INIA & & San Juan & Maynas & $12 / 2001$ \\
\hline 20 & Quistococha 2 & $03^{\circ} 48^{\prime} 45^{\prime \prime}$ у 73⒉'53" & San Juan & Maynas & $12 / 2001$ \\
\hline 21 & Mazán 1 - 2 & $03^{\circ} 29^{\prime} 34^{\prime \prime} \mathrm{y} 73^{\circ} 05^{\prime} 12^{\prime \prime}$ & Punchana & Maynas & $12 / 2001$ \\
\hline 22 & Indiana 1 & $03^{\circ} 29^{\prime} 57^{\prime \prime} \mathrm{y} 73^{\circ} 02^{\prime} 44^{\prime \prime}$ & Indiana & Maynas & $12 / 2001$ \\
\hline 23 & Lagunas 1 & $05^{\circ} 12^{\prime} 32^{\prime \prime}$ у 7539'43" & Lagunas & Alto Amazonas & $12 / 2001$ \\
\hline 24 & J. Herrera & $04^{\circ} 54^{\prime} 04^{\prime \prime}$ у 7340'13" & J. Herrera & Requena & $11 / 2002$ \\
\hline 25 & IIAP (Central) & $03^{\circ} 45^{\prime} 59^{\prime \prime}$ у 7316’30" & Iquitos & Maynas & $11 / 2002$ \\
\hline 26 & Lagunas 2 & $03^{\circ} 45^{\prime} 59^{\prime \prime}$ у $73^{\circ} 16^{\prime} 30^{\prime \prime}$ & Lagunas & Alto Amazonas & $11 / 2002$ \\
\hline 27 & Lagunas $3-4$ & $05^{\circ} 12^{\prime} 32^{\prime \prime}$ у 75³9'43" & Lagunas & Alto Amazonas & $11 / 2002$ \\
\hline 28 & Nauta-1 & $04^{\circ} 30^{\prime} 07^{\prime \prime}$ у 73³5'17" & Nauta & Loreto & $01 / 2003$ \\
\hline 29 & Olaya-1 & 04³4’30"y 7344’26" & Nauta & Loreto & $01 / 2003$ \\
\hline 30 & San Jorge-1 & 04³4’26”y 7343’39” & Nauta & Loreto & $01 / 2003$ \\
\hline
\end{tabular}


Anexo 2. Biometría de racimos de aguajes

\begin{tabular}{lrrrrrrrrr}
\hline Colección & $\begin{array}{r}\mathrm{N}^{\circ} \text { de } \\
\text { racimos } \\
\text { planta }\end{array}$ & $\begin{array}{r}\text { Longitud } \\
\text { pedúnculo } \\
\mathrm{cm}\end{array}$ & $\begin{array}{r}\text { Longitud } \\
\text { raquis } \\
\mathrm{cm}\end{array}$ & $\begin{array}{r}\mathrm{N}^{\circ} \text { de } \\
\text { raquillas }\end{array}$ & $\begin{array}{r}\text { Longitud } \\
\text { raquillas } \\
\mathrm{cm}\end{array}$ & $\begin{array}{r}\text { Frutos } \\
\text { por } \\
\text { raquillas }\end{array}$ & $\begin{array}{r}\text { Frutos } \\
\text { por } \\
\text { racimos }\end{array}$ & $\begin{array}{r}\mathrm{kg} \\
\text { frutos } \\
\text { racimos }\end{array}$ & $\begin{array}{r}\mathrm{kg} \\
\text { pulpa } \\
\text { racimos }\end{array}$ \\
\hline Quistococha 1 & 5.00 & 102.00 & 156.00 & 26.00 & 83.00 & 14.00 & 364.00 & 19.29 & 5.20 \\
Santo Tomás & 4.00 & 88.00 & 160.00 & 40.00 & 104.00 & 41.00 & 1640.00 & 86.92 & 23.42 \\
Santa Clara & 3.00 & 56.00 & 180.00 & 39.00 & 91.00 & 13.00 & 507.00 & 26.87 & 7.24 \\
Varillal & 3.00 & 80.00 & 162.00 & 36.00 & 101.00 & 10.00 & 360.00 & 19.08 & 5.14 \\
Quistococha 2 & 3.00 & 103.00 & 181.00 & 40.00 & 76.00 & 12.00 & 480.00 & 25.44 & 6.85 \\
Quistococha 3 & 6.00 & 114.00 & 154.00 & 41.00 & 115.00 & 27.00 & 1107.00 & 58.67 & 15.81 \\
Quistococha 4 & 4.00 & 111.00 & 160.00 & 40.00 & 69.00 & 7.00 & 280.00 & 14.84 & 4.00 \\
Quistococha 5 & 3.00 & 93.00 & 170.00 & 36.00 & 73.00 & 6.00 & 216.00 & 11.45 & 3.08 \\
Mazán 1 & 3.00 & 100.00 & 280.00 & 44.00 & 75.00 & 5.00 & 220.00 & 11.66 & 3.14 \\
Mazán 2 & 4.00 & 91.00 & 197.00 & 48.00 & 84.00 & 9.00 & 432.00 & 22.90 & 6.17 \\
Indiana & 6.00 & 70.00 & 190.00 & 48.00 & 84.00 & 6.00 & 288.00 & 15.26 & 4.11 \\
Jenaro Herrea & 5.00 & 120.00 & 240.00 & 38.00 & 115.00 & 18.00 & 684.00 & 36.25 & 9.77 \\
IIAP & 8.00 & 69.00 & 214.00 & 54.00 & 84.00 & 17.00 & 918.00 & 48.65 & 13.11 \\
Sumatoria & $\mathbf{5 7 . 0 0}$ & $\mathbf{1 1 9 7 . 0 0}$ & $\mathbf{2 4 4 4 . 0 0}$ & $\mathbf{5 3 0 . 0 0}$ & $\mathbf{1 1 5 4 . 0 0}$ & $\mathbf{1 8 5 . 0 0}$ & $\mathbf{7 4 9 6 . 0 0}$ & 397.29 & $\mathbf{1 0 7 . 0 4}$ \\
Máximo & $\mathbf{8 . 0 0}$ & $\mathbf{1 2 0 . 0 0}$ & $\mathbf{2 8 0 . 0 0}$ & $\mathbf{5 4 . 0 0}$ & $\mathbf{1 1 5 . 0 0}$ & $\mathbf{4 1 . 0 0}$ & $\mathbf{1 6 4 0 . 0 0}$ & $\mathbf{8 6 . 9 2}$ & $\mathbf{2 3 . 4 2}$ \\
Promedio & $\mathbf{4 . 3 8}$ & $\mathbf{9 2 . 0 8}$ & $\mathbf{1 8 8 . 0 0}$ & $\mathbf{4 0 . 7 7}$ & $\mathbf{8 8 . 7 7}$ & $\mathbf{1 4 . 2 3}$ & $\mathbf{5 7 6 . 6 2}$ & $\mathbf{3 0 . 5 6}$ & $\mathbf{8 . 2 3}$ \\
Mínimo & $\mathbf{3 . 0 0}$ & $\mathbf{5 6 . 0 0}$ & $\mathbf{1 5 4 . 0 0}$ & $\mathbf{2 6 . 0 0}$ & $\mathbf{6 9 . 0 0}$ & $\mathbf{5 . 0 0}$ & $\mathbf{2 1 6 . 0 0}$ & $\mathbf{1 1 . 4 5}$ & $\mathbf{3 . 0 8}$ \\
DesVest & $\mathbf{1 . 5 6}$ & $\mathbf{1 9 . 1 2}$ & $\mathbf{3 7 . 4 1}$ & $\mathbf{6 . 8 7}$ & $\mathbf{1 5 . 4 3}$ & $\mathbf{1 0 . 1 1}$ & $\mathbf{4 1 7 . 9 2}$ & $\mathbf{2 2 . 1 5}$ & $\mathbf{5 . 9 7}$ \\
Coef.Var. & $\mathbf{3 5 . 5 0}$ & $\mathbf{2 0 . 7 6}$ & $\mathbf{1 9 . 9 0}$ & $\mathbf{1 6 . 8 5}$ & $\mathbf{1 7 . 3 8}$ & $\mathbf{7 1 . 0 4}$ & $\mathbf{7 2 . 4 8}$ & $\mathbf{7 2 . 4 8}$ & $\mathbf{7 2 . 4 8}$ \\
\hline
\end{tabular}


Anexo 3. Biometrías de frutos y semillas

\begin{tabular}{|c|c|c|c|c|c|}
\hline \multirow[t]{3}{*}{$\mathrm{N}^{\circ}$} & \multirow[t]{3}{*}{ Ecotipo } & \multicolumn{4}{|c|}{ Medición $(\mathrm{cm})$} \\
\hline & & Largo & Ancho & Largo & Ancho \\
\hline & & Fruto $\mathrm{cm}$ & Fruto $\mathrm{cm}$ & Semilla cm & Semilla cm \\
\hline 01 & IIAP (Central) & 6.18 & 4.31 & 4.69 & 3.04 \\
\hline 02 & Indiana 1 & 5.00 & 4.22 & 3.73 & 3.13 \\
\hline 03 & Itaya 1 & 5.43 & 4.13 & 4.19 & 3.26 \\
\hline 04 & Itaya 2 & 3.68 & 2.53 & 3.23 & 2.39 \\
\hline 05 & Jenaro Herrera & 5.90 & 4.21 & 4.55 & 3.17 \\
\hline 06 & Lagunas 1 & 5.59 & 4.88 & 4.26 & 3.60 \\
\hline 07 & Mazán 1 & 5.20 & 4.00 & 4.15 & 3.04 \\
\hline 08 & Mazán 2 & 4.30 & 3.55 & 3.43 & 2.77 \\
\hline 09 & Quistococha 1 & 4.69 & 3.65 & 3.59 & 2.72 \\
\hline 10 & Quistococha 2 & 4.33 & 3.58 & 3.32 & 2.71 \\
\hline 11 & Quistococha 3 & 4.29 & 3.70 & 3.70 & 2.60 \\
\hline 12 & Quistococha 4 & 5.64 & 3.75 & 4.00 & 2.88 \\
\hline 13 & Quistococha 5 & 5.54 & 3.66 & 3.78 & 2.55 \\
\hline 14 & Samito & 5.56 & 4.39 & 3.81 & 3.30 \\
\hline 15 & Santa Clara & 6.34 & 3.62 & 4.07 & 2.72 \\
\hline 16 & Santa Rosa & 6.62 & 4.18 & 4.87 & 3.03 \\
\hline 17 & Santo Tomás & 4.09 & 3.58 & 2.87 & 2.66 \\
\hline 18 & Shiriara & 5.62 & 4.29 & 4.18 & 3.18 \\
\hline 19 & Varillal & 7.32 & 3.73 & 5.21 & 2.65 \\
\hline 20 & Yarina & 6.15 & 3.95 & 4.27 & 2.86 \\
\hline \multicolumn{2}{|c|}{ Máximo } & 7.32 & 4.88 & 5.21 & 3.60 \\
\hline \multicolumn{2}{|c|}{ Promedio } & 5.37 & 3.90 & 4.00 & 2.91 \\
\hline \multicolumn{2}{|c|}{ Mínimo } & 3.68 & 2.53 & 2.87 & 2.39 \\
\hline \multicolumn{2}{|c|}{ Des Vest } & 0.94 & 0.48 & 0.58 & 0.30 \\
\hline \multicolumn{2}{|c|}{ Coef Var. } & 17.40 & 12.29 & 14.42 & 10.42 \\
\hline
\end{tabular}


Anexo 4. Composición porcentual de 25 frutos y semillas de cada colección

\begin{tabular}{|c|c|c|c|c|c|c|c|c|c|c|}
\hline \multirow[t]{2}{*}{$\mathrm{N}^{\mathrm{o}}$} & \multirow[t]{2}{*}{ Colección } & \multicolumn{5}{|c|}{ Componente (gr) } & \multicolumn{4}{|c|}{ Porcentaje $(\%)$} \\
\hline & & Frutos & Semilla & Pulpa & Cáscara & $\begin{array}{l}\text { Epis- } \\
\text { perma }\end{array}$ & Semilla & Pulpa & Cáscara & $\begin{array}{r}\text { Epis- } \\
\text { perma }\end{array}$ \\
\hline 01 & IIAP & 1827.00 & 767.90 & 565.10 & 385.40 & 108.60 & 42.03 & 30.93 & 21.09 & 5.94 \\
\hline 02 & Indiana & 1513.50 & 661.50 & 375.20 & 371.30 & 105.50 & 43.71 & 24.79 & 24.53 & 6.97 \\
\hline 03 & Jenaro Herrera & 1625.00 & 796.60 & 446.30 & 279.40 & 102.70 & 49.02 & 27.46 & 17.19 & 6.32 \\
\hline 04 & Lagunas 1 & 2103.00 & 989.00 & 525.70 & 399.00 & 189.30 & 47.03 & 25.00 & 18.97 & 9.00 \\
\hline 05 & Lagunas 2 & 2230.38 & 958.50 & 510.62 & 435.38 & 325.88 & 42.97 & 22.89 & 19.52 & 14.61 \\
\hline 06 & Lagunas 3 & 2340.70 & 1081.00 & 747.90 & 350.00 & 161.80 & 46.18 & 31.95 & 14.95 & 6.91 \\
\hline 07 & Lagunas 4 & 1893.42 & 869.74 & 474.47 & 433.03 & 116.18 & 45.93 & 25.06 & 22.87 & 6.14 \\
\hline 08 & Mazán 1 & 1323.00 & 713.80 & 398.00 & 150.80 & 60.40 & 53.95 & 30.08 & 11.40 & 4.57 \\
\hline 09 & Mazán 2 & 879.60 & 492.80 & 221.80 & 101.20 & 63.80 & 56.03 & 25.22 & 11.51 & 7.25 \\
\hline 10 & Quistococha 1 & 913.10 & 453.20 & 244.60 & 146.80 & 68.50 & 49.63 & 26.79 & 16.08 & 7.50 \\
\hline 11 & Quistococha 2 & 862.20 & 380.00 & 207.90 & 201.20 & 73.10 & 44.07 & 24.11 & 23.34 & 8.48 \\
\hline 12 & Quistococha 3 & 900.50 & 447.50 & 165.50 & 209.50 & 78.00 & 49.69 & 18.38 & 23.26 & 8.66 \\
\hline 13 & Quistococha 4 & 1178.00 & 523.50 & 312.60 & 242.20 & 99.70 & 44.44 & 26.54 & 20.56 & 8.46 \\
\hline 14 & Quistococha 5 & 1044.70 & 449.60 & 328.80 & 203.30 & 63.00 & 43.04 & 31.47 & 19.46 & 6.03 \\
\hline 15 & Samito & 1441.00 & 662.85 & 417.90 & 288.20 & 72.05 & 46.00 & 29.00 & 20.00 & 5.00 \\
\hline 16 & Santa Clara & 1140.50 & 537.40 & 275.70 & 259.50 & 67.90 & 47.12 & 24.17 & 22.75 & 5.95 \\
\hline 17 & Santa Rosa & 2160.30 & 863.50 & 621.50 & 350.30 & 325.00 & 39.97 & 28.77 & 16.22 & 15.04 \\
\hline 18 & Santo Tomás & 729.60 & 325.80 & 167.20 & 137.40 & 99.20 & 44.65 & 22.92 & 18.83 & 13.60 \\
\hline 19 & Shiriara & 1407.25 & 699.50 & 311.25 & 281.00 & 115.50 & 49.71 & 22.12 & 19.97 & 8.21 \\
\hline 20 & Varillal & 1566.50 & 670.50 & 440.40 & 360.80 & 94.80 & 42.80 & 28.11 & 23.03 & 6.05 \\
\hline 21 & Yarina & 1407.50 & 652.50 & 407.50 & 270.00 & 77.50 & 46.36 & 28.95 & 19.18 & 5.51 \\
\hline \multicolumn{2}{|c|}{ Máximo } & 2340.70 & 1081.00 & 747.90 & 435.38 & 325.88 & 56.03 & 31.95 & 24.53 & 15.04 \\
\hline \multicolumn{2}{|c|}{ Promedio } & 1451.75 & 666.51 & 388.85 & 278.84 & 117.54 & 46.40 & 26.41 & 19.27 & 7.91 \\
\hline \multicolumn{2}{|c|}{ Mínimo } & 729.60 & 325.80 & 165.50 & 101.20 & 60.40 & 39.97 & 18.38 & 11.40 & 4.57 \\
\hline \multicolumn{2}{|c|}{ Des Vest } & 492.65 & 209.51 & 153.48 & 100.83 & 76.28 & 3.91 & 3.46 & 3.68 & 2.99 \\
\hline \multicolumn{2}{|c|}{ Coef Var } & 33.94 & 31.43 & 39.47 & 36.16 & 64.90 & 8.43 & 13.08 & 19.09 & 37.78 \\
\hline
\end{tabular}


Anexo 5. Evaluaciones de germinación de las colecciones

\begin{tabular}{lcccccccc}
\hline Procedencia & $\begin{array}{c}\text { Fecha } \\
\text { siembra }\end{array}$ & $\begin{array}{c}\text { Semillas } \\
\text { sembradas }\end{array}$ & $\begin{array}{c}\text { Inicio de } \\
\text { germinación }\end{array}$ & $\begin{array}{c}\text { Fin de } \\
\text { germinación }\end{array}$ & $\begin{array}{c}\text { Días de } \\
\text { germinadas }\end{array}$ & $\begin{array}{c}\text { Semillas } \\
\text { germinación }\end{array}$ & $\begin{array}{c}\text { \% } \\
\text { germinación germinación }\end{array}$ \\
\hline Samito & $08-03-00$ & 100 & $05-06-00$ & $10-09-00$ & 88 & 42 & 42.00 & Hipogea \\
Yarina & $08-03-00$ & 100 & $07-06-00$ & $10-09-00$ & 89 & 33 & 33.00 & Hipogea \\
Shiriara & $08-03-00$ & 100 & $12-06-00$ & $10-09-00$ & 94 & 38 & 38.00 & Hipogea \\
Itaya 1 & $15-09-00$ & 100 & $13-11-00$ & $30-03-01$ & 58 & 42 & 42.00 & Hipogea \\
Itaya 2 & $15-09-00$ & 100 & $13-11-00$ & $30-03-01$ & 58 & 61 & 61.00 & Hipogea \\
Santo Tomás & $15-05-01$ & 170 & $12-07-01$ & $26-11-01$ & 57 & 126 & 74.12 & Hipogea \\
Santa Rosa & $01-06-01$ & 110 & $31-07-01$ & $26-11-01$ & 60 & 97 & 88.18 & Hipogea \\
Santa Clara & $21-06-01$ & 110 & $14-08-01$ & $26-11-01$ & 53 & 63 & 57.27 & Hipogea \\
Quistococha 1 & $21-06-01$ & 110 & $15-08-01$ & $26-11-01$ & 54 & 57 & 51.81 & Hipogea \\
Varillal 1 & $10-07-01$ & 200 & $07-09-01$ & $26-11-01$ & 57 & 45 & 22.50 & Hipogea \\
Quistococha 2 & $23-07-01$ & 105 & $11-09-01$ & $26-11-01$ & 48 & 54 & 51.43 & Hipogea \\
Quistococha 3 & $23-07-01$ & 110 & $03-09-01$ & $26-11-01$ & 40 & 33 & 30.00 & Hipogea \\
Quistococha 4 & $31-07-01$ & 80 & $14-09-01$ & $26-11-01$ & 44 & 44 & 55.00 & Hipogea \\
Quistococha 5 & $31-07-01$ & 107 & $17-09-01$ & $26-11-01$ & 47 & 30 & 28.04 & Hipogea \\
Mazán 1 & $24-07-01$ & 55 & $25-09-01$ & $26-11-01$ & 61 & 5 & 9.09 & Hipogea \\
Mazán 2 & $24-07-01$ & 55 & $31-09-01$ & $26-11-01$ & 66 & 39 & 70.91 & Hipogea \\
Indiana 1 & $24-07-01$ & 55 & $31-09-01$ & $26-11-01$ & 66 & 35 & 63.64 & Hipogea \\
Lagunas & $21 / 10 / 01$ & 110 & $12-01-02$ & $29-04-02$ & 72 & 48 & 43.63 & Hipogea \\
Jenaro Herrera & $21 / 11 / 01$ & 100 & $15-02-02$ & $29-04-02$ & 75 & 26 & 26.00 & Hipogea \\
IIAP & $27 / 11 / 01$ & 125 & $12-02-02$ & $29-04-02$ & 72 & 62 & 49.60 & Hipogea \\
\hline
\end{tabular}

